\title{
SPS Gold Code Generation and Implementation for IRNSS User Receiver
}

\author{
Shachi Varku ${ }^{1}$, Swetha $\mathrm{A}^{2}$, Sharanya S Konandur ${ }^{3}$, Dileep D ${ }^{4}$, Aklpita L \\ Kulkarni ${ }^{5}$ \\ 1,2,3,5 (Department Electronics and Communication, Dr.Ambedkar Institute of Technology, Bangalore,India \\ ${ }^{4}$ (ISTRAC/ISRO, Bangalore, India
}

\begin{abstract}
The Indian Regional Navigational Satellite System (IRNSS) with Indian Constellation is an autonomous regional satellite navigation system developed by Indian Space Research Organization (ISRO). In this paper we have implemented the design of SPS gold code on MATLAB2012a and Xilinx ISE 14.7. The properties of SPS (standard positioning system) gold codes have been verified using MATLAB 2012a. It is found that IRNSS gold codes have three valued cross-correlation and four valued autocorrelation in line with theory. It is also found that IRNSS have balanced gold codes. This paper also deals with Hardware implementation of SPS Gold codes on Spartan-II FPGA Kit. The results are verified and analyzed with the PRN properties.
\end{abstract}

Keywords: IRNSS, PRN properties, SPS gold codes, ISRO, Xilinx

\section{INTRODUCTION}

The Indian Regional Navigation Satellite System (IRNSS) with an operational name of NAVIC is an independent regional satellite navigation system that is being set up by India, that will be used to provide accurate real-time positioning and timing services over India and the region extending to 1,500 kilometres around India. IRNSS will provide two levels of service, the standard positioning service will be open for civilian use, and a restricted service (an encrypted one) for authorized users (including the military). Both services will be carried on L5 (1176.45 MHz) and S band (2492.08 MHz).

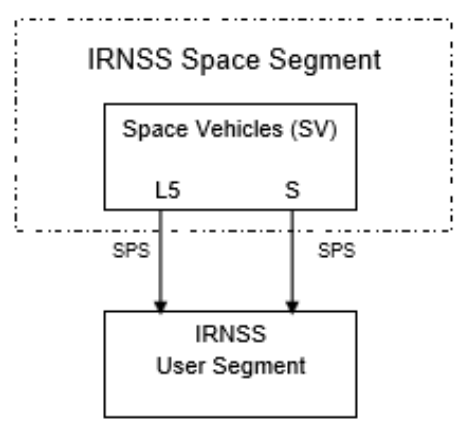

Fig 1. IRNSS Space Segment Interface with User Segment.

Figure 1 specifies the radio frequency interface between space and user segments. Each IRNSS satellite provides SPS signals in L5 and S bands.
The IRNSS consists of 3 segments: Space segment, ground segment and user segment. The satellites will be placed in two different orbital planes; 3 satellites in the Geostationary orbit (GEO) and 4 satellites in the Geo-synchronous orbit (GSO) with an inclination of $29^{\circ}$. The IRNSS constellation may be extended to eleven satellite constellation. The space segment is continuously monitored, controlled and managed by the ground segment.

User segment is related to user receivers in which "IRNSS SPS_GPS Receivers" will be used to receive IRNSS/GNSS signals. Based on various considerations the minimum number of satellites required for IRNSS constellation is worked out to be 7 ( 3 GSO and 4 IGSO). The 3 GSOs will be located at $32.5^{\circ} \mathrm{E}, 83^{\circ} \mathrm{E}$ and $131.5^{\circ} \mathrm{E}$ and the 4 IGSOs have their longitude crossings $55^{\circ} \mathrm{E}$ and $111.75^{\circ} \mathrm{E}$ (two in each plane). The primary objective of IRNSS is to achieve position accuracy of 20 meters $(2 \sigma)$ for dualfrequency users over India and the primary service area (a region extending to about 1,500 kilometers or 930 miles).

The section two provides proposed IRNSS user receiver, section-three of the paper provides the principle of positioning with IRNSS satellite. In Section four properties of PRN codes are discussed. In section five IRNSS gold code generation is discussed. In section six analysis of PRN properties is discussed. The section seven provides simulation and analysis results for SPARTAN 2 FPGA and this is followed by conclusion. 


\section{PROPOSED IRNSS USER RECEIVER}

The main objective of the IRNSS user receiver is to acquire signals simultaneously from all the IRNSS satellites using best satellites geometry of configured constellation(s) (w.r.t. GDOP \& Signal strength), provide the PNT(position, navigation and time) solution as outputs and display the same. The User Receivers shall have capability to receive IRNSS frequency

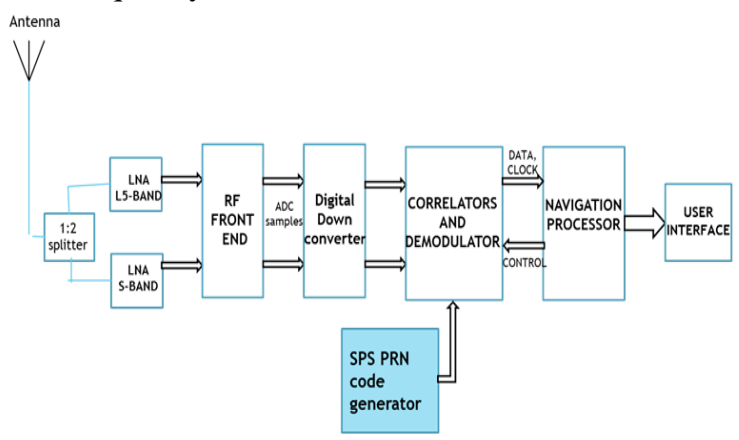

Fig.2 Block diagram for IRNSS user receiver bands (L5 \& S Bands).

Fig. 2 shows the block diagram of IRNSS user receiver which consists of antenna,L5 and $S$ band splitter, RF front end, digital down converter, correlators and demodulator, navigation processor, user interface and SPS code generator.

\section{THE PRINCIPLE OF POSITIONING WITH IRNSS SATELLITE}

Four satellites are required to provides accurate position.The largest tetrahedral is considered and are used to determine the position.

\subsection{Trilateration Principle}

The trilateration principle of operation is based on range measurements from signal sources, of which coordinates are known, to an unknown user position. Data from a single satellite pinpoints position to a large area of the earth's surface. Adding data from a second satellite narrows position down to the region where the two spheres overlap. Adding data from a third satellite provides a relatively accurate position and data from a fourth satellite - or more than four satellites - enhances precision. IRNSS has adopted a new, unique hybrid data structure with four subframes in its navigation messages. This data structure blends fixed format (Subframes 1 and 2 with the primary parameters clock terms, time, and ephemeris required for a position fix) and flexible format messages (Subframes 3 and 4 with the secondary parameters: ionospheric grid parameters, text messages, differential corrections, and so forth).

\subsection{Dilution of Precision (DOP)}

The position accuracy depends directly upon the respective satellite geometry. If the satellites are situated too close to each other, angular resolution deteriorates which, in turn, causes a greater positional error.

For this reason, the positioning space of satellites should be very large. To be able to make a statement about the current quality of a satellite geometry, the Dilution of Precision (DOP) is determined. A DOP of one thus corresponds optimal accuracy.

The effect of satellites geometry is usually expressed by the Geometric Dilution of Precision (GDOP) factor. The Dilution of Precision (DOP) is the ratio of the positioning accuracy to the measurement accuracy. It is usually greater than unity, however, if many satellites are observed (e.g. more than 8) the value of DOP can be less than unity.

\section{PROPERTIES OF PRN CODES}

The code sequence generated must satisfy the properties of PRN codes .

\subsection{Balance property}

Given a code sequence of length $\mathrm{L}$, the total number of ones in the sequence must not differ from the total number of zeros in the sequence by more than one, for every phase shift of the sequence [6].

\subsection{Run-length property}

A run is defined to be consecutive series of 1 's or 0 's within a sequence, and the run length is the number of 1's or 0 's in the series. In every sequence period the required distribution is that $1 / 2$ of the runs have a length of one, $1 / 4$ have a length of two, and so on [6].

\subsection{Autocorrelation property}

The autocorrelation function of code sequence must be two-valued. The autocorrelation value corresponding to zero shift, representing perfect correlation, should ideally be much larger than the other correlation value, which ideally should be as close to zero as possible [6] .

The autocorrelation function $\mathrm{r}(\mathrm{i})$ of any PN sequence of length $\mathrm{N}$ is given by [3].

$$
r(i)=\left\{\begin{array}{c}
1 \text { for } i=0 \\
-\frac{1}{N} \text { for }|i| \leq N-1
\end{array}\right.
$$




\subsection{Cross-correlation property}

Correlation is a measure of similarity between two sequences. When the two sequences compared are different it is the 'cross correlation' and when they are the same it is the 'autocorrelation'. [6] Mathematically, the correlation between two sequences $\mathrm{x}(\mathrm{k})$ and $\mathrm{y}(\mathrm{k})$ as a function of the time delay $\mathrm{m}$ is expressed as [3]

$$
R(m)_{x y}=\sum_{k=0}^{L-1} x(k) y(k+m)
$$

The cross correlation function is the number of agreements minus the number of disagreements as one code sequence is tested against a phase shifted version of the second sequence[6]

Correlation $=$ Total Agreements - Total Disagreements

\subsection{Welch bound}

The most commonly used bound on the cross correlation of sequences is Welch bound. The impact of this bound is that they dictate the limits within which all code sequence designs must lie. Thus it is not possible to independently design the correlation value and the set size, but it is necessary to allow the increase of the maximum absolute value of the correlation value in order to increase the set size for given sequence length.

Welch developed (1974) the lower bound of the cross correlation (normalized with respect to $\mathrm{N}$ ) between any pair of binary sequences of period $\mathrm{N}$ in the set of $\gamma$ sequences and is given by:

$$
\alpha_{\max } \geq \sqrt{\frac{\gamma-1}{N \gamma-1}}
$$

\section{IRNSS GOLD CODE GENERATION}

IRNSS utilizes Gold codes for the SPS signal. The codes are selected based on the autocorrelation and cross-correlation properties. In figure 3 the codes are generated using Linear Feedback Shift Registers.[2]

For SPS code generation, the two polynomials G1 and $\mathrm{G} 2$ are as defined below:

$$
\mathrm{G} 1: \mathrm{X} 10+\mathrm{X} 3+1
$$

$\mathrm{G} 2: \mathrm{X} 10+\mathrm{X} 9+\mathrm{X} 8+\mathrm{X} 6+\mathrm{X} 3+\mathrm{X} 2+1$

Polynomial G1 and G2 are similar to the ones used by GPS C/A signal. The G1 and G2 generators are realized by using 10 bits Maximum Length Feedback Shift Registers (MLFSR). The initial state of $\mathrm{G} 2$ provides the chip delay. G1 and G2 are XOR'ed for the generation of the final 1023 chip long PRN sequence. The time period of the PRN sequence is 1 millisecond.

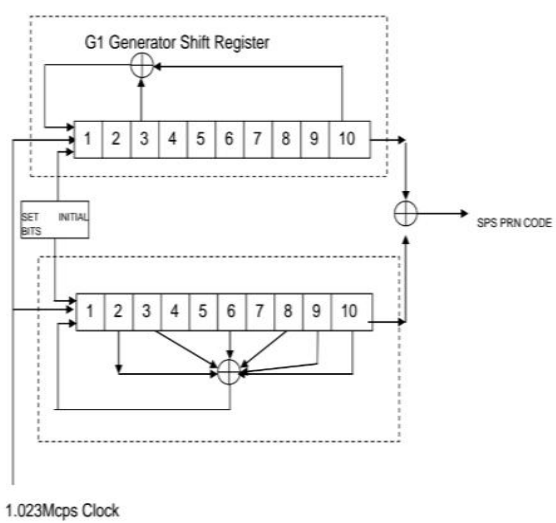

Fig. 3 SPS Gold Code Generator

\section{ANALYSIS OF PRN PROPERTIES}

Gold sequences form an important class of periodic sequences, which provide larger sets of sequences with good periodic cross-correlation. A set of Gold sequences of period $\mathrm{N}=2 \mathrm{n}-1$, consists of $\mathrm{N}+2$ sequences for which we have excellent cross-correlation properties.

\subsection{Cross-correlation property}

Cross-correlation function of gold sequence can only take one of the three different values defined for the preferred pair of sequence:

where, $\mathrm{t}(\mathrm{n})=1+2^{[(n+2) / 2]}$

A cross-correlation function taking these and only these values is called preferred three-valued crosscorrelation function and the corresponding pair of polynomials associated with it, is called a preferred pair of polynomials.[9] The analysis of crosscorrelation property of 7 satellites for both L5 band and $\mathrm{S}$ band is shown in table-1.

TABLE 1: The Tabulated Result Of 7 Satellites In L5 And S Band For Cross-Correlation Property Analysis

\begin{tabular}{|c|c|c|c|c|}
\hline \multicolumn{5}{|c|}{ CROSS CORRELATION } \\
\hline PRN ID & \multicolumn{2}{|c|}{ L5-BAND } & \multicolumn{2}{c|}{ S-BAND } \\
\hline 1 and 2 & Maximum Value & Minimum Value & Maximum Value & Minimum Value \\
\hline 1 and 3 & 0.0616 & -0.0635 & 0.0616 & -0.0635 \\
\hline 1 and 4 & 0.0616 & -0.0635 & 0.0616 & -0.0635 \\
\hline 1 and 5 & 0.0616 & -0.0635 & 0.0616 & -0.0635 \\
\hline 1 and 6 & 0.0616 & -0.0635 & 0.0616 & -0.0635 \\
\hline 1 and 7 & 0.0616 & -0.0635 & 0.0616 & -0.0635 \\
\hline 2 and 3 & 0.0616 & -0.0635 & 0.0616 & -0.0635 \\
\hline 2 and 4 & 0.0616 & -0.0635 & 0.0616 & -0.0635 \\
\hline 2 and 5 & 0.0616 & -0.0635 & 0.0616 & -0.0635 \\
\hline 2 and 6 & 0.0616 & -0.0635 & 0.0616 & -0.0635 \\
\hline 2 and 7 & 0.0616 & -0.0635 & 0.0616 & -0.0635 \\
\hline 3 and 4 & 0.0616 & -0.0635 & 0.0616 & -0.0635 \\
\hline 3 and 5 & 0.0616 & -0.0635 & 0.0616 & -0.0635 \\
\hline 3 and 6 & 0.0616 & -0.0635 & 0.0616 & -0.0635 \\
\hline 3 and 7 & 0.0616 & -0.0635 & 0.0616 & -0.0635 \\
\hline 4 and 5 & 0.0616 & -0.0635 & 0.0616 & -0.0635 \\
\hline 4 and 6 & 0.0616 & -0.0635 & 0.0616 & -0.0635 \\
\hline 4 and 7 & 0.0616 & -0.0635 & 0.0616 & -0.0635 \\
\hline 5 and 6 & 0.0616 & -0.0635 & 0.0616 & -0.0635 \\
\hline 5 and 7 & 0.0616 & -0.0635 & 0.0616 & -0.0635 \\
\hline 6 and 7 & 0.0616 & -0.0635 & 0.0616 & -0.0635 \\
\hline
\end{tabular}




\subsection{Autocorrelation property}

The autocorrelation function of a code sequence contains a single, large 'spike' at zero shift and a small, constant value for all other shifts.[6]. The analysis of autocorrelation property of 7 satellites for both L5 band and S band is shown in table-2.

TABLE 2: the tabulated result of 7 satellites in 15 and $\mathrm{s}$ band for Autocorrelation property analysis

\begin{tabular}{|c|c|c|c|c|c|c|}
\hline $\begin{array}{c}\text { PRN } \\
\text { ID }\end{array}$ & $\begin{array}{c}\text { L5-SPS Initial } \\
\text { Condition for } \\
\text { G2 Register }\end{array}$ & $\begin{array}{c}\text { Maximum } \\
\text { Value }\end{array}$ & $\begin{array}{c}\text { Minimum } \\
\text { Value }\end{array}$ & $\begin{array}{c}\text { S-SPS Initial } \\
\text { Condition for } \\
\text { G2 Register }\end{array}$ & $\begin{array}{c}\text { Maximum } \\
\text { Value }\end{array}$ & $\begin{array}{c}\text { Minimum } \\
\text { Value }\end{array}$ \\
\hline 1 & 1110100111 & 1 & -0.0635 & 0011101111 & 1 & -0.0635 \\
\hline 2 & 0000100110 & 1 & -0.0635 & 0101111101 & 1 & -0.0635 \\
\hline 3 & 1000110100 & 1 & -0.0635 & 1000110001 & 1 & -0.0635 \\
\hline 4 & 0101110010 & 1 & -0.0635 & 0010101011 & 1 & -0.0635 \\
\hline 5 & 1110110000 & 1 & -0.0635 & 1010010001 & 1 & -0.0635 \\
\hline 6 & 0001101011 & 1 & -0.0635 & 0100101100 & 1 & -0.0635 \\
\hline 7 & 0000010100 & 1 & -0.0635 & 0010001110 & 1 & -0.0635 \\
\hline
\end{tabular}

\subsection{Balance property}

In any gold code sequence, the number of $1 \mathrm{~s}$ is constantly one more than the number of $0 \mathrm{~s}$. [8] The analysis of balance property of 7satellites for both L5 band and S band is shown in table-3

TABLE 3: the tabulated result of 7 satellites in 15 and $\mathrm{s}$ band for balance property analysis

\begin{tabular}{|c|c|c|c|c|c|c|c|}
\hline $\begin{array}{c}\text { PRN } \\
\text { ID }\end{array}$ & $\begin{array}{c}\text { SV } \\
\text { Location } \\
\text { (degree) }\end{array}$ & $\begin{array}{c}\text { L5-SPS Initial } \\
\text { Condition for } \\
\text { G2 Register }\end{array}$ & $\begin{array}{c}\text { Number } \\
\text { of Ones }\end{array}$ & $\begin{array}{c}\text { Number } \\
\text { of } \\
\text { Zeroes }\end{array}$ & $\begin{array}{c}\text { S-SPS Initial } \\
\text { Condition for } \\
\text { G2 Register }\end{array}$ & $\begin{array}{c}\text { Number } \\
\text { of Ones }\end{array}$ & $\begin{array}{c}\text { Number } \\
\text { of } \\
\text { Zeroes }\end{array}$ \\
\hline 1 & $55 \mathrm{E}$ & 1110100111 & 512 & 511 & 0011101111 & 512 & 511 \\
\hline 2 & $55 \mathrm{E}$ & 0000100110 & 512 & 511 & 0101111101 & 512 & 511 \\
\hline 3 & $83 \mathrm{E}$ & 1000110100 & 512 & 511 & 1000110001 & 512 & 511 \\
\hline 4 & $111.75 \mathrm{E}$ & 0101110010 & 512 & 511 & 0010101011 & 512 & 511 \\
\hline 5 & $111.75 \mathrm{E}$ & 1110110000 & 512 & 511 & 1010010001 & 512 & 511 \\
\hline 6 & $32.5 \mathrm{E}$ & 0001101011 & 512 & 511 & 0100101100 & 512 & 511 \\
\hline 7 & $131.5 \mathrm{E}$ & 0000010100 & 512 & 511 & 0010001110 & 512 & 511 \\
\hline
\end{tabular}

\section{SIMULATION RESULTS AND ANALYSIS}

The PRN codes generation and analysis has been done using Matlab and Xlinx ISE simulations. Both Matlab and verilog codes have been used for the PRN code generation. The results are shown in the following figures.

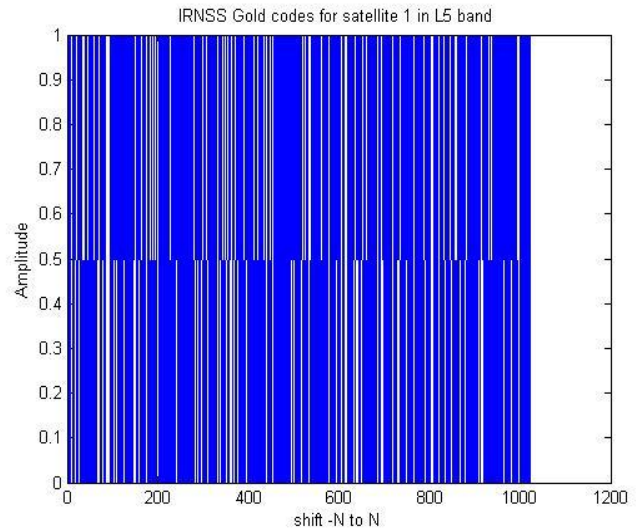

Fig 4 SPS Gold codes obtainof Satellite 1 in L5 band

Fig 4 shows the 1023 SPS gold code sequence generated for satellite 1 in L5 band using Matlab. The initial seeds are fed to G1 and G2 linear shift feedback registers, which differs for each satellite.

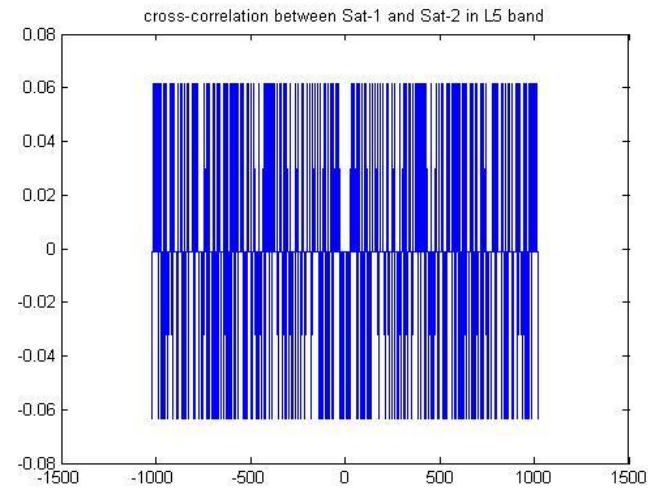

Fig 5a Crosscorrelation values of Satellite 1 in L5 band

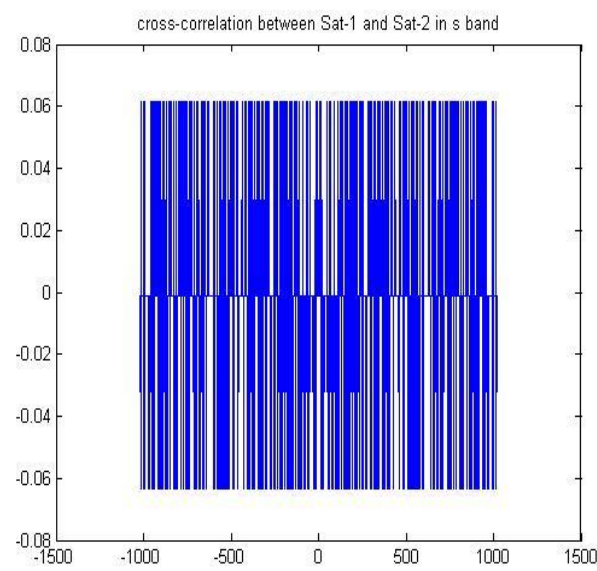

Fig $5 b$ Crosscorrelation values of Satellite 1 in $\mathrm{S}$ band

Fig. 5a,b shows the cross-correlation between two IRNSS gold code sequence. From the definition, the plot of the corresponding cross- 
correlation function is obtained with the matlab code shows that for this case $n=10$, we have $t(10)=$ $2^{6}+1=65$ and the only three values for the crosscorrelation function of the preferred Gold sequences will be: $\{-1,-65,63\}$ and after normalizing this three values we can see in Fig.3a and b, the crosscorrelation function values obtained correspond to the predicted ones.

Fig. 6a and $\mathrm{b}$ shows the auto-correlation of IRNSS gold code sequence. It is clear from the figure that the autocorrelation, large 'spike' is obtained at zero shift and a small, constant value for all other shifts in MATLAB.

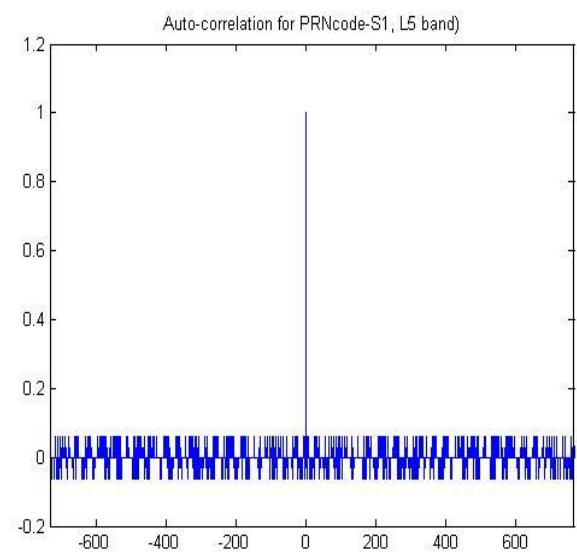

Fig. 6a Auto-correlation values of Satellite 1 in L5 band

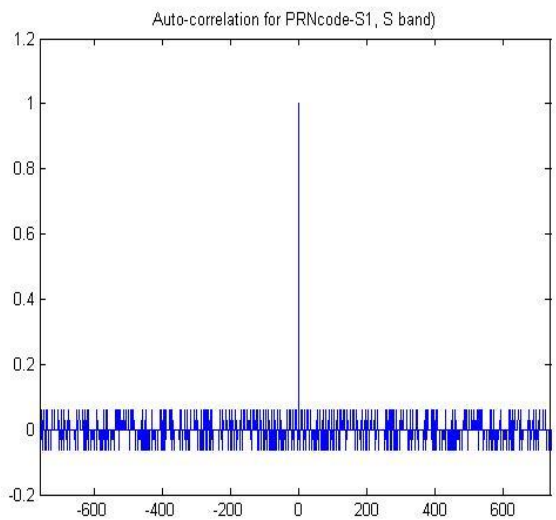

Fig.6b Auto-correlation values of Satellite 1 in L5 band

\subsection{Xilinx Implementation}

Fig. 7a demonstrates the generation of PRN code for Satellite-1 in L5 band. The output signal is used for validating the generated data bits carried out in Xilinx ISE 14.7 utilizing Verilog HDL. The output is obtained only when the respective count input is provided along with the reset input and clock input. Reset input is used as active high.

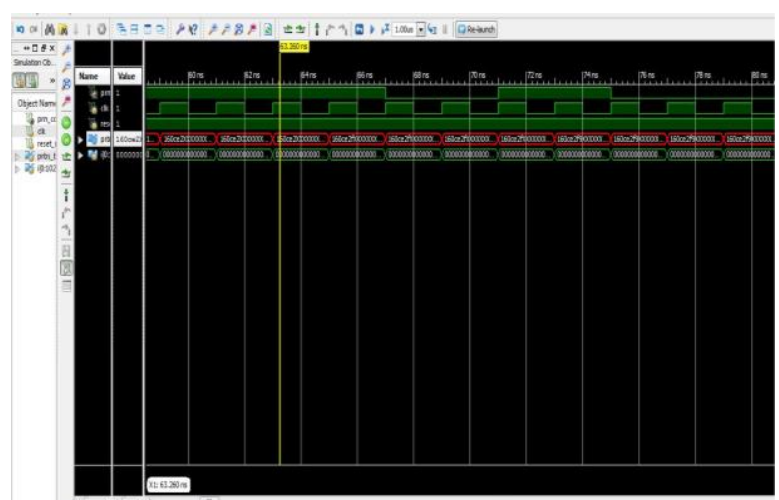

Fig.7a Xilinx simulation for Satellite 1 in L5 band

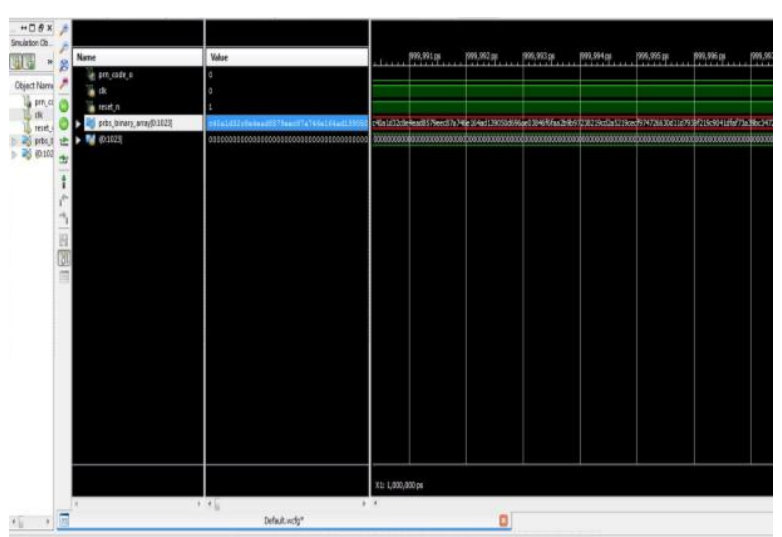

Fig.7b Xilinx simulation for Satellite 1 in $\mathrm{S}$ band

Figure $7 \mathrm{~b}$ shows the output signal for satellite 1 in $S$ band using Xilinx 14.7 and is validated with the ISRO setup.

\subsection{Hardware Implementation}

The SPARTAN-2 FPGA kit is used for PRN code generation in the hardware is represented in the Fig.8

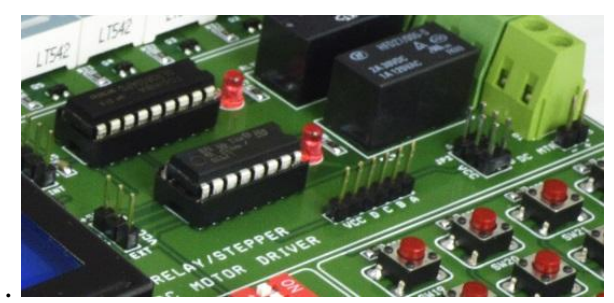

Fig.8 Spartan-2 kit

The push button switch provided enter key is utilized as the clock input. The clock was derived from the internal master clock. LED-1 is used for the displaying generated PRN data. 


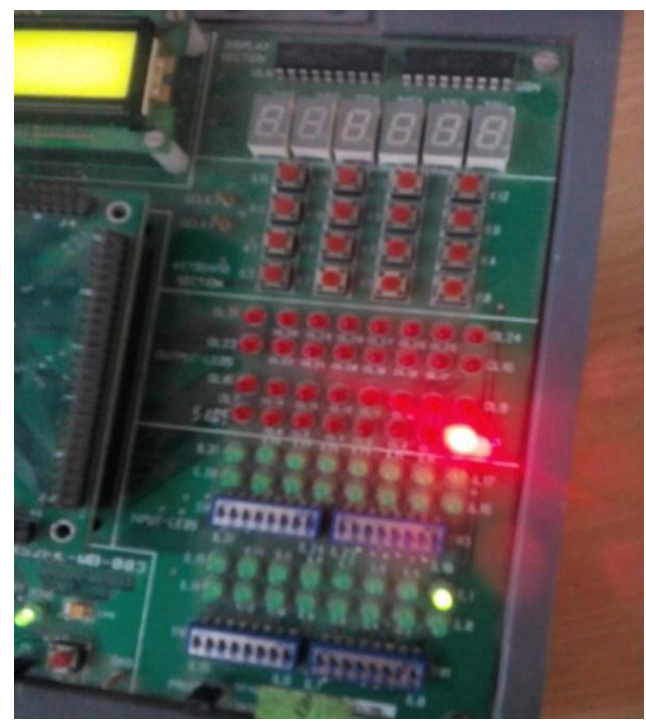

Fig 9 The Led Display Of The PRN Code Implementation.

Figure 8 shows the LED interfacing on the SPARTAN-2 FGPA kit using in built timer to show the SPS PRN codes generated.

\section{CONCLUSIONS}

In this paper we have generated and analyzed the properties of the PRN codes from the navigational system viewpoint. We have additionally done the design and implementation of PRN code on Spartan-II FPGA hardware. IRNSS receivers are implemented in either hardware or software. Due to flexibility and low cost, software receivers are preferred over the hardware. The design of PRN code on MATLAB2012a and Xilinx ISE 14.7 has been implemented and the properties of PRN codes have been verified using MATLAB 2012a.

\section{ACKNOWLEDGMENT}

This research was supported by ISRO and Dr.Ambedkar institute of technology. We are thankful to our guides Dileep D and Aklpita L Kulkarni who provided expertise that greatly assisted in the completion of the paper.

\section{REFERENCES}

[1]. R.C. Dixon Spread Spectrum System (2nd ed.)John Wiley and Sons, New York (1984), pp. 155-157.

[2]. ISRO IRNSS Signal in Space For Standard Positioning Service (version 1.0).

[3]. Akash B , Dileep D , Yashodha H, 'Generation and implementation of Pseudorandom codes for Navigation System in FPGA '(2016), India

[4]. A.R. Yashaswini, P. Siva Nagendra Reddy', G.N. Kodanda Ramaiah, H ,
'Generation and implementation of IRNSS Standard Positioning Signal', Kuppam Engineering College, Kuppam, Andhra Pradesh 517425, India (2016)

[5]. Richard Schwarz 'An Introduction to Linear Recursive Sequences in Spread Spectrum Systems' Filtronic Sigtek Inc. December 2001.

[6]. Mark W. Young, 'Design of a Gold Code Generator for Use in Code Division Multiple Access Communication System' University of Central Florida1985.

[7]. G. Hamza, A. Zekry, I. Motawie 'Implementation of a complete GPS receiver using Simulink IEEE Circuits Syst'. Mag, 9 (4) (2009), pp. 43-51

[8]. Abhijit Mitra 'On the Properties of Pseudo Noise Sequences with a Simple Proposal of Randomness Test' International Journal of Electrical, Computer, Energetic, Electronic and Communication Engineering Vol:2, No:9, 2008

[9]. Rodríguez-Henríquez, J.M. Rocha-Pérez, F. Amaro-Sánchez y F. Sandoval-Ibarra 'Generation of Gold-sequences With Applications to Spread Spectrum Systems'. 